\title{
Catálogo de colofones de manuscritos conservados en España (I)
}

\author{
Ma del Carmen Álvarez Márquez \\ Universidad de Sevilla
}

\section{Resumen}

Se recogen un total de treinta y seis colofones correspondientes a otros tantos manuscritos conservados en distintas bibliotecas de España. Un eslabón más de un proyecto de investigación que inicié hace algunos años, fruto del cual han sido tres artículos, y que tengo intención de proseguir, de ahí el número I que sigue al título, con la publicación de los que ya tengo recogidos y analizados.

Palabras clave: Historia del libro manuscrito; historia de la escritura, colofones; copistas; Diego de Anaya; Diego Gómez de Fuensalida; Conde de Haro; Conde de Gondomar; Duque de Osuna; Colegio de San Bartolomé de Salamanca; Francisco de Bruna.

\section{Colophons Catalog of Manuscripts in Spain (I)}

\section{Abstract}

In all, thirty-six colophon are collected here, which respectively correspond to an equal number of manuscripts preserved in different libraries in Spain. This is another stage in a research project which I undertook years ago and which has originated three articles. I intend to continue this project with the publication of those colophons already collected and analyzed, and hence the ordinal number "I" that follows the title.

Keywords: History of the manuscript book; history of writing, colophons; copyists; Diego de Anaya; Diego Gómez de Fuensalida; Conde de Haro; 
Conde de Gondomar; Duque de Osuna; Colegio de San Bartolomé de Salamanca; Francisco de Bruna.

El presente trabajo no es más que otra aportación a un proyecto de investigación, iniciado hace tiempo, con la finalidad de elaborar un censo lo más completo posible de copistas y manuscritos conservados en diferentes bibliotecas y archivos de España, cuya factura tuvo lugar entre el siglo XIII y el primer tercio del siglo XVI ${ }^{1}$.

En esta ocasión se han analizado treinta y seis, custodiados en la Biblioteca Nacional de España (BNE), Biblioteca Universitaria de Salamanca (BUSAL), Biblioteca del Palacio Real (BPR) y Fundación Lázaro Galdiano de Madrid (FLGM), que he agrupado en distintos epígrafes, según los datos proporcionados por los colofones.

1.- Manuscritos que consignan el nombre, oficio del copista, lugar y fecha de copia: 9.

- BNE: 6.

- BUSAL: 3.

2.- Manuscitos que consignan el nombre, oficio del copista y fecha de copia: 11

- BNE: 3.

- BUSAL: 7.

- BPR: 1.

${ }^{1}$ Coincidiendo la fecha con la que aparece en ellos, véanse mis trabajos: $\mathrm{M}^{\mathrm{a}}$ del Carmen ÁLVAREZ MÁRQUEZ, «Escribas y colofones en la Biblioteca Capitular y Colombina de Sevilla», Scribi e colofoni: le sottoscrizioni di copisti dalle origini all'avvento della stampa : atti del seminario di Erice. X Colloquio del Comité International de Paléographie Latine (23-28 ottobre 1993); a cura di Emma Condello e Giuseppe De Gregorio, Spoleto Centro Italiano di Studi sull'Alto Medioevo (1995), pp. 385-413; "Catálogo de los colofones de la Biblioteca Capitular y Colombina de Sevilla», Scriptorium, (XLIX , $\mathrm{n}^{\circ}$ 2, 1995), pp. 283-311; «La utilización de reclamos en diagonal en códices latinos escritos en el Reino de Castilla en el siglo XV», Scriptorium, (LIV, no 2, 2000), pp. 219-240; «El libro en la Baja Edad Media. Su caligrafía», coords. $\mathrm{M}^{\mathrm{a}}$ Encarnación Martín López y Vicente García Lobo, Las inscripciones góticas: II Coloquio Internacional de Epigrafía Medieval: León del 11 al 15 de septiembre 2006, León, Corpus Inscriptionum Hispaniae Mediaevalium 2010, pp. 263-332; y «Manuscritos de copistas hispanos (siglos XIV y primer tercio del XVI», coords. María Josefa Sanz Fuentes y Miguel Calleja Puerta, Paleografía II: las escrituras góticas desde 1250 hasta la imprenta. V Jornadas de la Sociedad Española de Ciencias y Técnicas Historiográficas, Oviedo, 18 y 19 de junio de 2007, Oviedo, Servicio de Publicaciones de la Universidad, 2010, pp. 51-106.

Titivillus, ISSN 2387-0915, ISSN-e 2603-9966, 2 (2016), pp. 9-26 
3.- Manuscritos que consignan el nombre del copista, lugar y fecha de copia: 16

- BNE: 7.

- BUSAL: 4.

- BPR: 4.

- FLGM: 1.

Cada ficha recoge los siguientes datos: noticias referidas específicamente a los diferentes encabezamientos, biblioteca donde se conserva y signatura, transcripción paleográfica de cada colofón, tipo gráfico o tipos gráficos utilizados para la copia del texto y, finalmente, procedencia.

Conclusiones:

- Los manuscritos analizados fueron copiados entre 1298 y 1487.

- Los lugares de copia se encuentran diseminados por toda España y cuatro se copiaron en el extranjero: Aviñón, «in castello Riuipiniani», en Roma «apud (S. Mariam) Rotondam» y en Montpellier.

- Las profesiones de los copistas que declaran su oficio son también variadas: escriba profesional (1); canónigos (2), uno de la Iglesia Catedral de Jaén y otro de la de Zamora; frailes (3), uno de ellos dominico y otro agustino, que se declara bachiller; mercader, habitante de la villa de Constantí cerca de Tarragona (1); notario y clérigo, alumno de Diego de Anaya, obispo de Cuenca (1); criado del contador real Alfonso González de León; alumno y familiar del doctor en Decretos y regente de la Cátedra de Prima de la Universidad de Salamanca Egidio García de Ontiveros (1); bachiller en Decretos del Colegio de San Bartolomé de Salamanca para la librería del citado colegio, otro el ya citado fraile agustino y el tercero sin ninguna otra precisión (3); escribano real (1); notario del Obispo de Cuenca (1); sacristán (1); clérigos (3), dos de ellos palentinos; librero (1); y, por último, boticario (1).

- Aunque no es frecuente, en ocasiones, se explicita el nombre de la persona a la que va destinado el códice. Así, el Ms. 10.133 de la BNE fue termiado el 13 de enero de 1385 en Aviñón por Álvar Pérez de Sevilla, canónigo de la Iglesia Catedral de Jaén, para Juan Fernández de Heredia, gran maestre de la Orden de San Juan del Hospital. Entre 1409 y el 4 de febrero del siguiente año, inicia Juan Fernánez, notario y clérigo, el Ms. 2.666 de BUSAL para su maestro Diego de Anaya, obipo de Cuenca, que terminó al año siguiente. Diego de Lombraña copió en 1434 el Ms. 2.208 de la BNE para su señor Alfonso González de León, contador real, en tanto que el Ms. 2.375 lo concluye el 11 de agosto de 1441 Juan de Capillas, bachiller en Decretos y colegial del Colegio de San Bartolomé de Salamanca para la biblioteca del citado colegio. Los manuscritos 2.313 y 2.439 de la BUSAL fueron copiados por Toribio Fernández, notario del obispo de Cuenca Diego de Anaya 
Maldonado, en tanto que el Ms. 2.183 de BUSAL lo fue por el sacristán Juan de «Valle Comitum» para Juan de Aranda. Asensio González concluyó el Ms. 9.247 de la BNE el martes 5 de junio de 1380 en la ermita de Santa María de Montemayor de Moguer para Vasco Gil de Tavira y Fernando de Cañamero el Ms. 12.085 lo terminó en Montpellier el 20 de noviembre de 1397 por comisión de su señor, Diego Gómez de Fuensalida, noble y religioso castellano, próximo a la figura de Fernando de Antequera durante el Compromiso de Caspe, que en estos momentos era abad de Santa María de Husillos, cerca de Palencia y luego de Valladolid, en tanto que, siendo obispo de Zamora, encargó en 1418 al canónigo Juan Alfonso el manuscrito con obras de Inocencio III, conservado en la BNE, procedente del Archivo Histórico Nacional.

- En julio de 1434 finaliza en la ciudad de Córdoba por comisión de Diego de Anaya Maldonado, en estos momentos obipo de Sevilla, un tal Resualio, parte del Ms. 2.667. El martes 1 de abril de 1477 concluye en Segura (Guipúzcoa) Juan García de Yarza el Lucidario conservado en la BPR para su maestro Juan Álvarez de Burgos, en tanto que Juan de Balaguer termina en Sevilla el Ms. 15.304 de la FLGM por encargo de Pero Afán de Ribera, del Consejo del Rey, para su primogénito, Fernando Gómez de Ribera, que contiene una obra muy utilizada a lo largo de toda la Edad Media e inicios de la Moderna destinada a la formación de príncipes y nobles, De regimine principum de Egidio Romano en su versión castellana. Queda, finalmente, por reseñar el Pontificale copiado en Tarazona en 1487 por Fernando Gómez de Ágreda a expensas y para uso personal de Andrés Mártínez Ferriz, obispo de Tarazona.

- Los tipos gráficos utilizados, como no podía ser de otro modo, pertenecen al ciclo de las escrituras góticas, con predominio de la cursiva y la híbrida, utilizándose la littera textualis formata o en su variedad rotunda como escritura publicitaria y, en alguna ocasión, también para el texto y, en este caso, capitales y unciales agrandadas como publicitaria o letras quebradas como el Amen del Ms. 2.666 de la BUSAL.

- Finalmente y en cuanto a la procedencia: uno tuvo como anterior propietario al Duque de Osuna; otro a Diego Monfar y Sors; dos al Conde de Haro; cinco al Colegio de San Bartolomé de Salamanca, portando algunos el escudo de Diego de Anaya y Maldonado; otro procede del Archivo Histórico Nacional; dos del Convento de Santo Tomás de Ávila; otro de la Biblioteca del Conde de Gondomar, portando en el f. 1v: «Este libro es de Gonzalo de Monrroy» y en el margen superior del f. 89v: «Del Obispo de Ábila o Quenca»; otro perteneció al Monasterio de 
Uclés, pasando luego al Archivo Histórico Nacional, adonde también pasó un manuscrito de la Catedral de Ávila; a la Biblioteca del Colegio de Cuenca perteneció otro que porta el ex libris real de la época de Carlos IV-Fernando VII; tres manuscritos de la BUSAL, uno de ellos forma parte de la presente relación, presentan rúbricas autógrafas de Alfonso de Palencia y firmas de M. Martínez y Dr. Forcada; uno procede de la Casa Profesa de la Compañía de Jesús de Sevilla, pasando luego a la biblioteca de Francisco de Bruna, siendo el Lucidario, conservado en la BPR, es el que más notas de propiedad porta: f. $1 \mathrm{r}$ de guarda: De Francisco de Salvatierra; Diego Díaz; f.1v de guarda: «Esta fize yo Juan Ynniges de Segura, anno de 1556 annos, echa en la leal ciudad de Murcçia»; Don Pedro de Arrieta; ex libris de Alfonso XIII en tinta roja.

Terminan aquí las noticias extraídas del análisis de los treinta y seis manuscritos recogidos en este trabajo, pasando ahora a las fichas cátalográficas de cada uno de ellos, siguiendo los apartados indicados más arriba.

\section{Manuscritos que consignan el nombre, oficio del copista, lugar y fecha de copia}

1.- 1331, marzo, 27, miércoles. [Valencia]. ANTHONIUS MARTI, SCRIPTORIS CIUIS VALENÇIE.

BNE. Ms. 73. FRANCESC EIXIMENIS. Llibre dels angels.

f. 306v: Iste liber fuit expletus / per manus Anthoni Marti,/ scriptoris ciuis Valençie,/ die mercurii, vicesima / septima mensis marcii, / anno $\mathrm{M}^{\circ}$ CCCC XXXI ${ }^{\circ}$.

Tipo gráfico: Híbrida fuera de sistema tipo K, ya que las a presentan copete; $s$ finales sigmáticas; iniciales por trazar y mayúsculas rellenas de amarillo.

2.- 1385, enero, 13. Aviñón. ALUAR PEREZ DE SEUILla, CANONIGO EN LA CATHEDRAL YGLESIA DE IAHEN, DE MANDADO DE DON IOHAN FERNANDES DE EREDIA, POR LA GRAÇIA DE DIOS, DE LA SANCTA CASA DEL ESPITAL DE SANT IOHAN DE / IERUSALEM.

BNE. Ms 10.133. FERNÁNDEZ DE HEREDIA, Juan (1308?-1395). Grant Cronica de Espanya primera partida.

f. $605 \mathrm{v}, 2^{\mathrm{a}}$ col:: Aqui fenesçe la primera partida / de la Grant Cronica de Espanya, com/pilada de diuersos libros e ystorias / por el muy reuerent en Christo, Padre / e Senyor, don Iohan Fernandes de / Eredia, por la graçia de Dios, de la Sancta / Casa del Espital de Sant Iohan de / Ierusalem, maestro humil e aguar/dador de los pobres de Christo,/ la qual cronica, de mandado del / dicho senyor, yo, Aluar Perez de / Seuilla, canonigo en la Cathedral / Yglesia de Iahen, escreui de mi propria / mano et fue acabada en Aui/nyon, a 
XIII dias del mes de ie/nero el anyo del Nasçimiento de / Nuestro Senyor M CCC $^{\circ}$ E LXXX $^{a} V^{\circ}$./ DEO / GRATIAS./

Tipo gráfico: Littera textualis en su variedad rotunda y como escritura publicitaria capitales y unciales agrandadas.

Procedencia: Duque de Osuna

3.- 1387. Castillo de Santo Domingo (Zaragoza). FRATER ALFOnSUS DE SANCTO GERUASIO.

BUSAL. Ms. 1882. Códice facticio con diversos tratados de Lógica.

f. 181r: Frater Alfonsus de Sancto Geruasio

f. 269v: Iste liber est ad vsum fratris Alfonsi de Sancto-Ger/uasio prouincie Sancti Iacobi, quod partim scripsit et partim fe/cit seribi Cesaraguste, prouincie Aragonie, et fuit / perfectus in Crastrum (sic) Dominici, patris Ordinis Predicatorum, / anno Domini $\mathrm{M}^{\circ} \mathrm{CCC}^{\circ} \mathrm{LXXX} \mathrm{VH}^{\circ}$.

Tipo gráfico: Gótica cursiva de trazos descabalgados

4.- 1401, mayo, 4, miércoles. Barcelona. RAYMUNDUS IANUARII, MERCATOR, HABITATOR VILLE SEU CASTRI DE CONSTANTINO, DIOCESIS TERACHONENESIS.

BNE. Ms. 8.242. [VALERIO MÁXIMO. Hechos y dichos memorables, traducidos al catalán por fray Antonio Canals].

f. 240v, $2^{\mathrm{a}}$ col: Iste liber expletus fuit per manus / Raymundi Ianuarii, mercatoris, / habitatoris ville seu castri de Cos/tantino, diocesis Terachonensis, die / mercurii, quarta mensis madii, anno / a Natiuitate Domini millessimo $\mathrm{CCCC}^{\circ}$ primo et / fuit perfectus in ciuitate Bachinone.

Tipo gráfico: Típica bastarda cursiva de buen trazado.

Procedencia: Diego Monfar y Sors.

5.- 1409 - 1410, febrero, 4. Poveda de la Obispalia (Cuenca) IOHANNES FERNANDI, NOTARIUS AC CLERICUS, ALUMPNUS DOMINI DIDACI [DE ANAYA], EPISCOPI CONCHENSIS.

BUSAL. Ms. 2.666. BOECIUS. De consolatione philosophiae [cum expositione Nicolai Treveti].

f. 120r, $2^{a}$ col.: Finito libro sit / laus gloria Christo./ Qui scripsit scribat et / semper cum Domino uiuat./ Uiuat in terris magis / in celis qui scripsit nomine felix./ Viuat prudenter gazas / habeat sapienter./

120 r-v: A línea tirada: Hic est liber scriptus, ideo semper sit Deus benedictus. AMEN./

Iste liber de venerabili eximio et domino $<$ meo $>$ domino Didaco, episcopo Conchensi, est, qui ego, de predicto domino episcopo Iohannes Fernandi alumpnus, scripsi et in anno a Natiuitate Domini $\mathrm{M}^{\circ}$ CCCC IX ${ }^{\circ}$ incepi et quarta die mensis febroarii in predicto / rure de Poueda, quod de supradicto meo episcopo est, et in anno Domini $\mathrm{M}^{\circ} \mathrm{CCCC}^{\circ} \mathrm{X}$ compleui, qui Boecius De consolacione / vocatur et cum glosa et testo contendit. Et ego, supradictus Iohannes Fernandi, ob reuerencia beatissi//me gloriose semperque Uirginis

Titivillus, ISSN 2387-0915, ISSN-e 2603-9966, 2 (2016), pp. 9-26 
Marie et cum adiutorio Dei et predicti domini mei episcopi, quem Deus pro / sua pietate per longitudinem dierum permanere faciat in suo episcopatu, feci et scripsi et compleui, ut scrip/tum atque predictum est, et numquam in istum librum nullam pennam cisi, nisi due penne cum quibus scribe/re incepi et primitum liber iste perfectus fuit que ille fuerunt perfecte in tempore isto./

Iohannes Fernandi, notarius ac clericus./ Pennula scriptoris quiescit fessa laboris./

Tipo gráfico: Littera textualis formata de dos módulos (texto y glosa) y bastarda cursiva para el colofón. El Amen del colofón con letras quebradas.

Procedencia: [Colegio de San Bartolomé de Salamanca]

6.- 1418 [Zamora]. JOHANNES ALFONSI, CANONICUS.

BNE. Ms. 8.854. INOCENCIO III, PAPA. De miseria hominis.- Proverbia Senecae cum commentariis. - De octo vitiis principalibus. - De sacrificio Missae.

f. 65r: De mandato reuerendissimi / in Christo patris ac domini domini / Didaci, episcopi Zamorensis, ego Jo/hannes Alfonsi, suus canonicus, / scripssi istum librum, anno / Domini millesimo quadrigentesimo / decimo octauo./

f. 157v: De mandato reuerendissimi in Christo patris / ac domini domini Didaci, episcopi Za/morensis, ego Johannes Alfonsus, canonicus,/ scripsi istum librum anno Domini / millesimo quadrigentesimo decimo octauo.

Tipo gráfico: Bastarda cursiva.

Procedencia: Archivo Histórico Nacional (9-1-B).

7.- 1434. Sevilla. DIEGO DE LONBRANNA, CRIADO DE ALFONSO GONÇÁLEZ DE LEÓN, CONTADOR DEL REY, POR MANDADO DEL DICHO ALFONSO GONÇÁLEZ.

BNE. Ms. 2.208. VALERIO MÁXIMO. Hechos y dichos memorables, traducción en castellano de la que hizo al catalán fray Antonio Canals, según Nicolás Antonio. Mutilado al comienzo. Nota en el margen superior del f. $1 \mathrm{r}$ de Iriarte: «Historia de los Romanos, traducida por el Gran Cardenal».

f. $227 \mathrm{v}, 1^{\mathrm{a}}$ col.: Finito libro laus sit et gloria Christo./

[E]ste libro de Valerio Maximo escriuio Diego / de Lonbranna, criado de Alfonso Gonçalez de / Leon, contador del Rey, por mandado del dicho $\mathrm{Al} /$ fonso Gonçalez, en la muy noble çibdat de Seuilla, / anno del Sennor de M $\mathrm{CCCC}^{\circ} \mathrm{XXX} \mathrm{IIII}^{\circ}$ annos.

Tipo gráfico: Híbrida fuera de sistema tipo $\mathrm{C} / \mathrm{H}$ : muchas $l$ no presentan bucles, $d$ unciales, $s$ finales sigmáticas. Alzados de muchos de los primeros renglones del folio o de los capítulos alargados.

8.-1438, agosto, 29. Universidad de Salamanca. ALFONSSUS GARSSIE, ALUPNUS AC FAMILLIARIS DOMINI EGREGIII DOCTORIS EGIDII GARSSIE DE FONTIUEROS, DECRETORUM DOCTORIS, REGENTIS TUNC CATHEDRAM PRIME IN CIUITATE SALAMANTINA, SCRIPSSIT. 
BNE. Ms. 7.536. BALDUS DE UBALDIS. Lectura super Decretalibus Innocentii IV. (inicio mutilado) (1v-62r). - JOHANNES CALDERINI. Quaestiones $\left(62 \mathrm{v}-143 \mathrm{r}, 1^{\mathrm{a}}\right.$ col.).-ANGELUS DE UBALDIS DE PERUSIO. Consilia (143v-152v).-ANÓNIMO. Repetitio super C 2 q. 6 Decreti Gratiani.

f. 1r, 1 ${ }^{\mathrm{a}}$ col.: Explicit oppus excellentissimi domi /domini (sic) Baldi de Perusio, vtriusque ju/ris doctoris, conpositum super Innocen/cio./ Deo gratias. Amen./

Alfonssus Garssie, alupnus ac fa/milliaris domini egregiii doctoris / Egidii Garssie de Fontiueros, Decre/torum doctoris, regentis tunc ca/thedram Prime in ciuitate Sala/mantina scripssit./ Alfonssus Garsie / de Segobia, scriptor (rúbrica).

f. 102r, $1^{\text {a }}$ col.: Expliciunt consilia domini Baldi de / Perusio, legum doctoris, cuius anima in / pace requiescat. Amen./ Et fuerunt scripta et perfecta per do/minum Alfonssum Garssie, familiarem / domini Egidii Garssie, Decretorum doctoris, / regentis cathedram prime Canonum / in scolis maioribus in ciuitate Salaman/tina, anno Domini millesimo quadrigen/tesimo tricesimo octauo, vicessima no/na die menssis augusti. Alfonssus / Garssie, scriptor./ Alfonssus / Garssie (rúbrica)

f. 143r, $1^{\text {a }}$ col.: Expliciunt consilia domini Angeli / de Perusio, excelentissimi legum doctoris, / que fuerunt scripta per dominum [anulado con puntitos debajo: An/gelum de Perusio] Alfonssum Garssie de / Segobia, familiarem egregii domini / Egidii / Garssie de Fontiueros, Decretorum doctoris,/ regentis cathedram prime Canonum / in scolis maoribus in alma Vniuersitate / ciuitatis Salamantine. Alfonssus Gar/ssie (rúbrica).

En el margen inferior del f. 37r: Alfonssus Garssie (rúbrica)

Tipo gráfico: Gótica cursiva de pequeño módulo con tendencia a la bastarda en los caídos y alzados de $b, l, f, s$ y littera textulis formata como escritura publicitaria.

Procedencia: Convento de Santo Tomás de Ávila.

9.- 1441, agosto, 11. [Salamanca]. JOHANNES DE CAPILLAS, IN DECRECTIS BACHALARIUS ISTIUS COLLEGII SANCTI BARTHOLOMEI COLLEGIALIS, PERFECI SCRIBERE ISTUM LIBRUM PRO LIBRERÍA DICTI COLLEGII.

BUSAL. Ms. 2.375. PETRUS DE ANCHARANO. [Commentarius in tertium librum Decretalium].

f.175r, 2 ${ }^{\mathrm{a}}$ col-175v, $1^{\mathrm{a}}$ col.: Deo gracias/ Et ego Johannes de Capillas, in $\mathrm{De} /$ crectis bachalarius istius $\mathrm{Col} /$ legii Sancti $\mathrm{Ba}<\mathrm{r}>$ tholomei collegi/alis, perfeci scribere istum librum // pro librería dicti Collegii, anno Domini $\mathrm{M}^{\circ}$ CCCC XLI, die vero XI augusti, et quia / transumptum erat viciosum / rogo michi parcatur et iste liber corrigatur./ Iesus.

Tipo gráfico: Gótica cursiva de módulo muy pequeño, por lo que puede ser calificada como notular, y littera textualis formata como escritura publicitaria y en el colofón. Inicial principal por trazar.

Procedencia: Escudo de Diego de Anaya custodiado por un ángel en f. 1r, $1^{\text {a }}$ col.

Titivillus, ISSN 2387-0915, ISSN-e 2603-9966, 2 (2016), pp. 9-26 
Manuscritos que consignan el nombre, oficio del copista y fecha de copia

1.- 1386. GONÇALO RODRIGUEZ DE SANTIAGO, ESCRIUANO DEL REY. BNE. Ms. 12.722. LIVIO, Tito. Tercera década.

f. CCXVIIIv, $2^{a}$ col. Aquí se acaba este libro. Iesus.

A línea tirada: En el nonbre de Dios, Padre e Fijo e Espiritu Santo, aquí se acaba esta terçera parte de / Titus Liuius, en la qual se contiene de la conquista que los romanos fezieron a el rey Fe/lipo de Maçedonia e Anabit, rey de Laçedemonia, e al poderoso rey de Antiochia e / a Hanibal e a los Etholianos e a otros muchos reyes e principes del mundo, contra los quales ouie/ron muy esçelentes vitorias e cuenta fasta la muerte de Anibal e de los Sçipiones e de los / otros prinçipes, el qual escriuio Gonçalo Rodriguez de Santiago, escriuano del Rey, en la era de mille / e quatroçientos e quaçientes veynte e quatro annos, reynante en Castilla e en Leon, / el muy alto e muy poderoso prinçipe, nuestro sennor, el rey don Juan, que Dios mantenga./

Gonçalo (rúbrica)

Tipo gráfico: Híbrida: $a$ sin copete y $s$ finales sigmáticas, que en el colofón permuta por una cursiva caligráfica, y la littera textualis formata artificiosa, adornada con rasgueos de pluma y de trazado epigráfico, como escritura publicitaria.

Procedencia: Conde de Haro.

2.- 1411, marzo, 27. TORIBIUS FERDINANDI, NOTARIUS DICTI DOMINI EPISCOPI CONCHENSIS [DE MANDATO DOMINI MEI AC PATRIS REUERENDI DOMINI DOMINI DIDACI, EPISCOPI CONCHENSIS] (a su mano se debe también el ms. 2.439 de BUSAL).

BUSAL. Ms. 2.313. Códice misceláneo

f. 124r: Perficitur Vincentius Ystorial de mandato do/mini mei ac patris reuerendi domini domini Didaci, / episcopi Conchensis, quia sua mercede de tenebris / ad lucem, de aduersis ad prospera, de paupertate ad diuicias, de / nichilo ad aliquod, de bono ad melius me dignatus est perdu/cere Deus retributor bonorum, qui neminem uult perire tri/buat sibi uitam et gloriam sempiternam suamque personam in prosperi/tate continua sospitate longena gaudio et leticia / sua clementi misericordia conseruare dignetur. Amen/ Anno Domini millesimo $\mathrm{CCCC}^{\circ} \mathrm{XI}^{\circ}$ videlicet XXVII die mensis marcii./

Toribius Ferdinandi, notarius dicti domini episcopi Conchensis (rúbrica).

f. 197r: Benedictus sit Deus et laudabilis et gloriosus per cuncta seculorum secula. Amen. /

Quis scripsit scribat semper cum Domino uiuat. Amen. Toribius Ferdinandi uo/catur a Domino benedicatur./

Scribere qui nescit non putat esse labore./

Tres digiti scribunt et cetera membra dolent/ 
Scriptum fuit a To/ribio Fernandi, anno / Domini M ${ }^{\circ}$ CCCC XI, XXVII die mensi marcii./ Toribius Fernandi, / notarius episcopalis (rúbrica)./

Tipo gráfico: Gótica cursiva con clara tendencia a la bastarda, trazada con una pluma de tallo ancho. Iniciales por trazar

Procedencia: Manuscrito hecho por encargo de Diego de Anaya. Colegio de San Bartolomé de Salamanca.

3.- 1438-1441, enerO. FRATER JOHANNES DE MONLEON, ORDINIS FRATRUM PREDICATORUM.

BUSAL. Ms. 2.061. Códice facticio.

ff. 225r-234v. PETRUS DE FRIAS. Orationes academicae $(1438,1439)$.

f. 227v: Explicit principium quod conposuit et fecit Petrus de Frias, in Artibus bachalarius, / in die Sancti Luche, Studio Sallamantino, anno Domini $\mathrm{M}^{\circ}$ $\mathrm{CCCC}^{\circ} \mathrm{XXXXVIII}^{\circ}$, / et ipsum scripssit frater Johannes de Monleon, Ordinis Fratrum Predicatorum, anno $\mathrm{M}^{\circ} 41^{\circ}$.

f. 234v: Explicit principium quod fecit Petrus de Frias, in Artibus bachalarius, die Sancti Luche / in Studio Sallamantino, anno Domini millesimo quadringentessimo / XXXIX ${ }^{\circ}$, quod scripssit Monleon, anno XXXX primo, / mense ianuarii.

Tipo gráfico: Bastarda cursiva y littera textualis formata como escritura publicitaria. Iniciales principales por trazar.

4.- 1451, marzo, 27. JOHANNES DE VALLE COMITUM, SACRISTA, PER TE JOHANNES / DE ARANDA SCRIPSIT EUM ET PERFECIT.

BUSAL. Ms. 2.183. 1r-83v. Repertorium iuris civilis domini Baldi et aliorum doctorum.

f. $83 \mathrm{v}, 1^{\mathrm{a}}$ col.: Explicit repertorium domini Baldi / et alliorum doctorum excellenti/ssimorum et cetera per te Johannes / de Aranda scripsit eum et perfecit / XVII ${ }^{a}$ die mensis marci, anno / Domini millesimo $\mathrm{CCCC}^{\circ} \mathrm{LI}^{\circ}$. Deo gracias.

ff. 84r-154v: Repertorium super operibus Bartholi de Saxoferrato sive lux caecorum et requies doctorum

f. $154 \mathrm{v}, 1^{\mathrm{a}}$ col.: Qui scripsit scribat et semper cum domino / uiuat. Johannes de Valle Comitum uo/catur a Christo benedicatur. Amen./ Johannes, sacrista (rúbrica).

Tipo gráfico: Hỉbrida de pequeño módulo con $s$ finales sigmáticas y littera textualis formata muy bien ejecutadas con una bella $I$ quebrada en el f. $84 \mathrm{r}$ : Incipit.

5.- 1456, junio, 15, martes. JOHANNES DE TORQUEMADA, [CLERICUS PALENTINE DIOCESIS].

BUSAL. Ms. 2.372. JOHANNES DE PLATEA. Super IV libros Institutionum Iustiniani.

f. $182 \mathrm{v}, 2^{\mathrm{a}}$ col. Hic liber perfectus fuit per me, Johannem de Torquemada, die / martis, XV menssis iunii, anno Domini millessimo CCCCLVI ${ }^{\circ}$./

Deo gracias./

Titivillus, ISSN 2387-0915, ISSN-e 2603-9966, 2 (2016), pp. 9-26 
Johannes de / Torquemada (rúbrica)

Tipo gráfico: Híbrida con $s$ finales sigmáticas, típica notular, y littera textualis formata y unciales como escrituras publicitarias.

Procedencia: Escudo de Diego de Anaya en f. 1r, timbrado por el capelo cardenalicio.

6.- 1457, enero, 9. JOHANNES DE TORQUEMADA, CLERICUS PALENTINE DIOCESIS.

BUSAL. Ms. 2.278. NICOLAUS DE TUDESCHIS. Lectura in primum librum Decretalium.

f. 146v: Perfectus fuit / iste liber IX die messis (sic) ianuarii, anno Domini / millessimo CCCCLVII per me, Johannem de Torquemada,/ clericum Palentine diócesis.

Tipo gráfico: Híbrida con $s$ finales sigmáticas, típica notular, y littera textualis formata como escritura publicitaria. Gran número de abreviaturas y pequeño módulo.

7.- 1461. FRATER ALFONSUS DE SANCTO MARTINO, ORDINIS EXIMII GLORIOSI AUGUSTINI NECNON BACHALARIUS.

BUSAL. Ms. 2.132. Códice facticio.

ff. 1r-192v. PAULUS VENETUS. Expositio in libros Posteriorum Aristotelis.

f. 192r: Explicit opus subtile Posteriorum scriptum ac editum a reuerendissimo / magistro ac egregio doctorum magistro Paulo de Veneciis et per me, fratrem / Alfonsum de Sancto Martino, Ordinis eximii gloriosi Augustini necnon bachalarium,/ anno Domini $\mathrm{M}^{\circ} \mathrm{CCCC}^{\circ} \mathrm{LXI}^{\circ}$.

Tipo gráfico: Híbrida, tipo $\mathrm{C} / \mathrm{H}$, con $s$ finales sigmáticas y $d$ unciales con bucles. Libro para uso personal, como lo denota la escritura poco cuidada y las tachaduras.

8.- 1462, marzo, 1. IOHANNES DE VELGARA, BACHALARIUS.

BNE. Ms. 2.207. BARTOLUS DE SAXOFERRATO. De inquisitionibus. - BALDUS DE UBALDIS. Lectura super $V$ libro Codicis.- Rubrica super iudices.- PETRUS DE ANCHARANO. Repetitio iuris canonici.- BARTHOLOMAEUS DE SALICETO. Repetitio iuris. - BRTOLUS DE SAXOFERRATO. Liber testimoniorum.NICHOLAUS DE SICILIA, ABBAS. Arbor consanguinitatis.- De regulis iuris fragmentum. f. $67 \mathrm{v}, 1^{\mathrm{a}}$ col: Et sic est finis huius llectionis dicti domini / Baldi de Perussio quinto li/bro. Deo gratias, amen, et intacte Eius Virgi/nis Marie, Matri, amen. Perfecta fuit dicta opera prima die marci ano (sic) a Natiuitate Domini / $\mathrm{M}^{\circ}$ CCCC $^{\circ}$ LXII pro bachalario Iohanni de Velgara. Virgo Dei Maria felici n/omine digna ora pro famullis, o Benedicta, tuis.

Tipo gráfico: Iniciales por trazar. Littera textualis fracturada como escritura publicitaria y gótica cursiva de pequeño módulo como escritura de texto con los alzados del primer renglón quebrados. Desde el inicio al f. 83v la misma mano. Gran número de notas marginales. 
9.- 1468, mayo, 10. CRISTOFOL BOSCH, LIBRATER.

BNE. Ms. 4.023. [Condestable D. PEDRO DE PORTUGAL. Sátira de felice e infelice vida].

f. LXXIIr: Fou acabad lo present libre a / X de mag any 1468 de ma / den Cristofol Bosch, librater./ Deo gratias.

Tipo gráfico: Bella littera textualis tipo $E$, ya que las a no presentan copete, de dos módulos: texto y glosa, que en muchos folios no se copió.

10.- 1469-1470. PETRUS ORTIZ, CLERICUS.

BUSAL. Ms. 1877. Códice misceláneo

f. 300v: Escriptus fuy anno Domini $\mathrm{M}^{\circ} \mathrm{CCCC}^{\circ} \mathrm{LXX}$ Petrus Ortiz

f. 301r: En este libro son copilados onze tratados:...

Este libro se acabo anno Domini $\mathrm{M}^{\circ}$ CCCC LXIX. Petrus Ortiz, clericus

Tipo gráfico: Híbrida con $s$ finales sigmáticas.

11.- 1471, diciembre. JUANES DE MAYORICA, BOTICARIO.

BPR. Ms. II/3.063. Libro de recetas en castellano y Secretos de Medicina de don Juan Enrriquez:

f. 73r, $1^{\mathrm{a}}$ col.: Este libro es acabado, miercoles / a (espacio en blanco) del mes de dezienbre del / anno del Sennor de mill e quatro/çiento e setetenta (sic) e vn annos./

Este libro me fezid Juanes de / Mayorica, boticario.

Siguen tres recetas en una escritura muy cursiva y descuidada

Tipo gráfico: Híbrida con $d$ unciales, con y sin bucles y $s$ finales sigmáticas, por lo que puede ser calificada de híbrida $\mathrm{C} / \mathrm{H}$ y littera textualis formata como escritura publicitaria.

Procedencia: Biblioteca del Conde de Gondomar. En 1v: «Este libro es de Gonzalo de Monrroy» y en el margen superior del f. 89v: «Del Obispo de Ábila o Quenca».

\section{Manuscritos que consignan el nombre del copista, lugar y fecha de copia}

1.- 1298, diciembre. Uclés. PETRUS FERRANDUS ACHARAÇENSIS.

BNE. Ms. 924. Biblia sacra (v. III Vetus Testamentum) (mutilada al comienzo y al final).

f. 122r, $2^{\mathrm{a}}$ col.: Ista Biblia fuit scripta Vclensi et / perfecta in mensse deçembris, anno / Domini millesimo duçentesimo nonage/simo octauo et era millesima tre/çentesima triçesima sexta, regnante / in Castella et in Legione rege Ferdi/nando qui cepit regnare in etate $\mathrm{XI}^{\text {cim }} /$ annorum cum magno labore quia erant / contra ipsum rex Aragonie et rex Gra/nate et multi barones sui regni, / et fuit incepta in tempore prioris fratris / Iacobi et perfecta in tempore Munionis, / prioris Vclensem (sic), et frater Dominicus / cum esse sacrista fecit eam incipere / et dum esset subprior fecit eam

Titivillus, ISSN 2387-0915, ISSN-e 2603-9966, 2 (2016), pp. 9-26 
perficere. / Qui me scripsit scribat et semper cum Domino / uiuat. Petrus Ferrandus Acharaçensis me / scribsit; ipse sit benedictus per cuncta secula. Amen./

f. 122v, al margen de la primera columna: Yo, Garçes Perez, / la fiz por / mandado de / frey Miguel.

$\mathrm{Al}$ colofón precede una nota cronológica desde Adán, ambos con una escritura de menor módulo que el resto del manuscrito. Tercer volumen de una Biblia sacra en cuatro (Ms.922/925), todos muy deteriorados y mutilados.

Tipo gráfico: Littera textualis en su variedad rotunda.

Procedencia: Monasterio de Uclés, después Archivo Histórico Nacional.

2.- 1365. Monasterio de los Predicadores de Valencia. BENEDICTUS FABREGUES.

BNE. Ms. 9.018. ARISTÓTELES. Opera. (Muy deteriorado y mutilado).

f. 143r, 1 ${ }^{\text {a }}$ col.: Istud opus fere totum scripsit Benedictus Fabregues / in Monesterio Predicatorum Valencie et mul/tis cum laboribus et quam plurimis erumpnis finem / ad usque prope produxit anno millesimo $\mathrm{CCC}^{\circ} \mathrm{LX}^{\circ}$ quin/to, de cuius finem glorificetur Altissimus, qui pasci/tur inter ther choros sanctoum, per infinita / seculorum secula. Amen./ Iesus. Maria. Bernardus. Tomas.

$2^{a}$ col.: Vt gaudere solet fessus iam nauta labore/

Desiderata diu littora nota videns, /

Haud aliter scriptor obtato finem libelli /

Exultat viso, lassus ipse quidem /

Scribere qui nescit nullam putat esse laborem /

Tres digiti scribunt sed ceterea membra dolent.

Tipo gráfico: Gótica cursiva.

Procedencia: Convento de Santo Tomás de Ávila.

3.- 1380, junio, 5, martes. Ermita de Santa María de Montemayor (Moguer). ASENSIO GONZALEZ PARA VASCO GIL DE TAVIRA.

BNE. Ms. 9.247. Crónica de los monjes, con diversos dichos de Santos Padres y otros documentos.

f. 96v, 2a col.: Et yo, Asensio Gonzalez, lo / escriui para Vasco Gil de Tauira, / e acabose en Santa Maria de Monte/mayor, çerca de Moguer, martes,/ çinco dias de junio, era de / mil e quatroçientos e diez e ocho / annos, a loor del Padre e del Fijo e / e (sic) del Spiritu Santo, tres personas e vn Dios/ verdadero./

(Otra mano): f. 110v, $1^{\text {a }}$ col.: Acabado es el libro, lo/ado sea Iesuchristo. El libro / es acabado, Dios sea loado por / syempre. Amen. Yo, muy grant / pecador, que este libro escreui, ru/ego al que lo leyere que ruegue / a Dios por mi, que El, por la su / Pasyon, me faga con los sus / santos en el çielo morador. A/MEN./

Qui me scripsit scribat e sen/per cum Domino biuat. Amen./

Titivillus, ISSN 2387-0915, ISSN-e 2603-9966, 2 (2016), pp. 9-26 
f. $115 \mathrm{v}, 1^{\mathrm{a}}$ col.: Yo pecador, que este libro escreui,/ ruego al que lo leyeres que ru/egue a Dios por mí, que me de / gracia que acabe en su seruiçio en / penitençia verdadera. Porque / la mi anima aya gualardon de / vida perdurable en el su rego (sic) celestial por siempre. Amen./ Otrosy por el que lo mando escre/uir, que Dios lo cumpla de uirtu/des, porque acaba en su serui/çio. Amen. Dezid el Pater / Noster con el Aue Maria, que Dios lo cum/pla por ruego de la Uirgen Glori/osa Santa Maria. Amen./

Quy me scripsit escribat / et senper cum Domino biuat./ Amen.

Tipo gráfico: Típica escritura precortesana. Hibrida fuera de sistema tipo $\mathrm{K} / \mathrm{H}$ : $a$ con y sin copete, $s$ finales sigmáticas.

Procedencia: Conde de Haro.

4.- 1397, noviembre, 20. Montpellier. FERNANDUS DE CANNAMERO DE MANDADO NOBILIS ET POTENTIS VIRI ET DOMINI MEI, DOMINI DIDACI GOMECII DE FUENTE SALIDA, ABBATIS DE FUSELLIS.

BNE. Ms. 12.085. PAULUS GUILLELMUS. Lectura super Clementinas.

f. 184r, 2a col.: Explicit lectura Pauli Guillelmi / et Geucellini super Clementinas./

Finito libro gratias refferamus Christo./

Qui scripsit scribat ac semper cum Domino / viuat. Viuat in celis Fernandus de / Cannamero nomine felix./ De mandado nobilis et potentis viri et / domini mei, domini Didaci Gomecii de Fuente Salida,/ abbatis de Fusellis, ego Fernandus de $\mathrm{Ca} /$ nnamero hunc librum scripssi in Montopesu/lane compleuique die vicesima menssis / nouenbris anno Domini millesimo trecente/simo nonagesimo séptimo, pontificatus / sanctissimi in Christo patris et domini nostri domini Bene/dicti diuina prouidencia pape terciidecimi / anno quinto./

Tipo gráfico: Gótica cursiva de pequeño módulo con rasgos de la bastarda. Gran número de abreviaturas e iniciales por trazar. Littera textualis en su variedad rotunda como escritura publicitaria.

Procedencia: Catedral de Avila (Sig.: Av. 25) y Archivo Histórico Nacional (Sig. AHN. 3-1-B)

5.- 1415, enero. [Cartuja de Portaceli de Valencia]. ALUARUS SALAMANTINUS. BNE. Ms. 870. [Breviarium Ordinis Cartusiensium].

f. 16r: Et nota quod in mense ianuarii / anno a Natiuitate Domini $\mathrm{M}^{\circ} \mathrm{CCCC}^{\circ}$ / $\mathrm{XV}^{\circ}$ quod istud fuit scriptum in/cepit currere littera C.

f. CCXCIIr: Finito collectario sit laus et gloria Christo. Amen. Scribens / sit beatus cum sanctis asociatus. Scripsit domnus Aluarus Sala/mantinus, cui Deus conferat sua bona. Amen./

Tipo gráfico: Littera textualis en su variedad rotunda, con algunas $l$ prolongadas y con rasgueos de pluma en el primer renglón.

6.- 1434, julio. Córdoba. RESUALI REUERENDI DOMINI DIDACI, ARCHIEPISCOPI YSPALENSIS, PRECEPTU [DIEGO DE ANAYA MALDONADO]. 
BUSAL. Ms. 2.667. (ff. 1r-57r) TELESPHORUS DE CUSENTIS. Libellus de causis, statu, cognitione ac fine presentis scismatis et tribulationum futurarum.- $(58 \mathrm{r}-60 \mathrm{v})$ BERNARDUS CLARAEVALLENSIS. Epistola ad Raymundum de cura et modo rei familiaris.

f. 60v: Mense julii, anno a Nativitate Domini $\mathrm{M}^{\circ} 434^{\circ}$, scripsit Resualio / opus istud reuerendi domini Didaci, archiepiscopi Yspalensis, / preceptu, in civitate Cordubense. Deo gratias. Amen./

f. 2v: Dominus michi adiuctor non timebo quid faciat micho homo, Domine non avertas faciem tuam a me in quacumque die tribulor, inclina ad me aurem tuam, Domine Iesu Christe filii vivi misere mei, amen.

Tipo gráfico: Bastarda cursiva y littera textualis formata como escritura publicitaria. Iniciales por trazar.

Procedencia: Diego de Anaya.

7.- 1444, enero, 14. Salamanca. DIDACUS ISPALENSIS.

BUSAL. MS. 2.452. BALDUS DE UBALDIS. [Lectura super sexto libro Codicis]. f. $347 \mathrm{v}, 1^{\mathrm{a}}$ col.: Finito libro sit infinita / laus et gloria Domino Christo./ Explicit lectura domini Baldi / super $\mathrm{VI}^{\mathrm{O}}$ libro Codicis scripta per / Didacum Ispalensem pro fa/mossissimo in Legibus ba/chal (raspado y escrito encima:) ario et egregio viro / Johannes (espacio en blanco) in / ciuitate Salamantense, anno / a Natiuitate Domini millesimo / quadringentesimo qua/dragesimo quarto [anulada con puntos debajo: decima] / alias sextodecima quarta / menssis januarii, Deo / gratias et eius Genitrici. Amen./

Tipo gráfico: Híbrida fuera de sistema, tipo $\mathrm{H} / \mathrm{E}$, ya que las $f$ y $s$ unas veces descienden bajo la línea de escritura y otras no, y $s$ finales sigmáticas, de módulo muy pequeño, y littera textualis formata como escritura publicitaria. Iniciales por trazar. Sin duda fue un manuscrito escrito por encargo, en concreto para un bachiller en Leyes, en este caso en la ciudad de Salamanca, de nombre Juan.

8.- 1445, mayo, 24. Ajofrín (Toledo). ALFONSUS SEGOBIENSI.

BNE. Ms. 4.035. GUIDO DE MONTE ROTERIO. Manipulus curatorum.

f. 208r, 2a col:: DEO GRATIAS. HIC / liber scriptus fuit a me, Alfonso / Segobiensi in loco de Aiofrin, de man/dato domini fratris Alfonsi de Sancto Mar/tino, sacrosancte Theologie pre/sentatus, de Ordine Fratruum (sic) Predi/catorum, et perfectus fuit nono $\mathrm{ka} / \mathrm{lendas}$ iunii anno a Natiuitate Domini / millessimo quatuorcentessimo quadrigen/tessimo quinto. Qui scripsit scri/bat semper cum Domino uiuat. Amen

Tipo gráfico: Gótica cursiva con tendencia a la bastarda y textualis fracturada como escritura publicitaria.

9.- 1463, junio, 10. In castello Riuipiniani. SILUESTRE BARI DE FABRICA. BPR. Ms. 1.948. PSEUDO-ARISTÓTELES. De moralibus et regimine dominorum, qui alio nomine dicitur de decretis secretorum.

f. 174: Finit opus Aristotilis de secretis / secretorum. Laus Deo./ 
Scriptum fuit per me, Siluestrem / Bari de Fabrica in Castro Riuipini/ani, anno Domini 1463 mensis juniii / die X finiui pontificatus domini nostri / Pii pape secundi, indictione XI.

f. [3]v: Scriptum fuit per me Siluestrem Bari de Fabrica in castello Riuipiniani anno Domini 1463 mense junii die X.

Tipo gráfico: Hibrida con $s$ finales sigmáticas que se hace cada vez más descuidada

Procedencia: De la Biblioteca del Colegio de Cuenca (f. 1r). Ex libris real de la época de Carlos IV-Fernando VII.

10.- 1465, febrero, 10. Roma apud (S. Mariam) Rotondam. IACOBUS DE ALEMANIA BASSA.

BUSAL. Ms. 21. Códice misceláneo.

ff. 1r-152v: CYRILLUS ALEXANDRINUS. Thesaurus adversus haereticos.

f. 152v: Per Iacobum de / Alemania Bass $<\mathrm{a}>$ finitus anno $\mathrm{LXV}^{\circ}, \mathrm{X}^{\circ}$ die mensis februarii Rome apud / Rotondam et sic est finis, sit laus et gloria trinis./

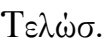

Tipo gráfico: Hibrida con $s$ finales sigmáticas y $a$ con y sin copete

Procedenia: Rúbricas autógrafas de Alfonso de Palencia y firmas de M. Martínez y Dr. Forcada en f. 1v. Igual en los manuscrtos 63 y 95: rúbricas, correcciones y anotaciones margianales de Alfonso de Palencia y firmas de M. Martínez y Dr. Forcada en f. 1 r.

11.- 1469, septiembre, 26. Salamanca. DIDACUS MAZQUEFFA.

BUSAL. Ms. 2.204. [PETRUS ABOVES, OFM. Super libros Sententiarum].

f. 108 r, $1^{a}$ col.: Deo / gracias./ Finita fuit ista recollectio Salaman/tice, quam ego, Didacus Mazqueffa, / propria manu finiui anno a Natiuita/te Domini $\mathrm{M}^{\circ}$ CCCC $^{\circ}$ LXVIIII $^{\circ}$, die / martis, XXVI septembris. / Didacus Mazqueffa.

Tipo gráfico: Escritura que muestra ya rasgos de la humanística cursiva, con arranques característicos de los alzados de las letras desde la izquierda, $d$ unciales con caídos de su segundo trazo hacia la izquierda, utilizando la littera textualis formata como escritura publicitaria y una bella $D$ quebrada en Deo gracias.

12.- 1477, abril, 1, martes. Segura (Guipúzcoa). IOHAN GARÇIA DE YARÇA, [EL QUAL ESCRIUI PARA MI MAESTRO JUAN ALUAREZ DE BURGOS].

BRP. II/793. Lucidario. Libro que ordenó el rey don Sancho, de esclaresçida memoria, fijo del muy noble rey don Alfonso e dela noble reyna doña Violante, su muger, el qual fue el sereno rey de los rreyes que fueron en Castilla que ovieron nonbre don Sancho, el qual libro ordeno por arte de Filosofia natural e de Estrologia e de Teologia que trata en los capitulos siguientes.

f. CCXVIIIr-v: Finito libro sit laus gloria Christo./

Benedicamus Domino Deo, gratias./

Et yo, Machin de Yarça, fijo de Iohan Garçia de / Yarça, escriui este libro, Dios sea loado, en la / villa de Segura de Guipuzcoa, martes, primero / dias

Titivillus, ISSN 2387-0915, ISSN-e 2603-9966, 2 (2016), pp. 9-26 
del mes de abril anno del nasçimiento del Nuestro / Saluador Iesu Christo de mille e quatroçientos e / setenta e siete annos, el qual escriui para mi ma/estro Juan Aluarez de Burgos en dozientas e / diez e ocho fojas. E porque es verdat puse / aquí mi nonbre. Machin (rúbrica).

CCXVIIIr: (de otra mano en el margen inferior) Conçertado. Loado sea Dios (rúbrica)

f. CCXVIIIv: (margen inferior) son erradas XXXIII fojas así son de tornar a escreuir / LXVI fojas

Tipo gráfico: Escritura cortesana.

Procedencia: De Francisco de Salvatierra. Diego Diaz (f. 1r de guarda). Esta fize yo Juan Ynniges de / Segura, anno de 1556 annos, e/cha en la leal ciudad de Murçia (f. 1v de guarda). Don Pedro de Arrieta. Ex libris de Alfonso XIII en tinta roja.

13.- 1478, febrero, 6, viernes. Barcelona. PAULUS OLIUERII MINORICENSIS. BNE. Ms. 1.984. [FRANCESC EIXEMENIS. Llibre de les dones].

f. 287r, $2^{\mathrm{a}}$ col.: Finito libro sit laus gloria Christo./ Qui scripsit scribat semper / cum Domino viuat. Amen./

Quod fuit perfectum scribendi per / me Paulum Oliuerii Minoricensis in/sule degens in ciuitate Barchinone / die veneris $\mathrm{VI}^{\mathrm{O}}$ die febroarii anni $\mathrm{M}^{\mathrm{I}} / \mathrm{CCCC}^{\mathrm{I}}$ LXX ${ }^{1}$ octaui./

Tipo gráfico: Empieza utilizando una textualis tipo T/E al presentar dos tipos de $a$, con y sin copete, no bien ejecutada, que termina en una escritura muy ligada y de trazado cursivo y la littera textualis formata con rasgueos de pluma como escritura publicitaria.

14.- 1480. Sevilla. JUAN DE BALAGUER [YO, PERO AFAN / DE RIBERA, DEL CONSEJO DEL REY,/ NUESTRO SENNOR, MANDE FAZER / ESTE LIBRO PARA MI FIJO FERNAN / GOMEZ DE RIBERA].

FLGM. Ms. 15.304. EGIDIO ROMANO. Regimiento de los principes e sennores que han de regnar los otros, traducido del latín al romance por fray Juan García de Castrojeriz de la Orden de los Frailes Menores, confesor de la Reina de Castilla para el infante don Pedro.

f. $274 \mathrm{r}-\mathrm{v}, 2^{\mathrm{a}}$ col. $-\mathrm{-}^{\mathrm{a}}$ col.: ... E porque en este libro, / que es dicho Regimiento de / príncipes, son contenidas mu/chas doctrinas prouechosas / a la uida humana, yo, Pero Afan / de Ribera, del Consejo del Rey,/ nuestro señor, mande fazer / este libro para mi fijo Fernan / Gomez de Ribera e que lo haya / por mayoradgo con los otros bienes míos, segund / mi ordenaçión, a fin que los que // de mi desçendieren decoren / sus personas e asy paresçien/do a sus progenitores uirtu/oso merescan ser honrrados./ Escriuiolo Juan Balaguer / en Seuilla en el anno del nas/cimiento del Sennor de mil / y quatrocientos y ochenta / annos, reynantes los muy / altos e muy esclaresçidos / principes reyes e sennores / don Fernando e donna Ysabel,/ reyes de Castilla e de Leon e / de Aragon.

Tipo gráfico: Littera textualis en su variedad rotunda. Bella inicial ornamentada y escudo de armas al final de la segunda columna en el margen inferior del f. $1 \mathrm{r}$.

Titivillus, ISSN 2387-0915, ISSN-e 2603-9966, 2 (2016), pp. 9-26 
15.- 1486, diciembre, 15. Chinchilla [de Montearagón] (Albacete) MARCO DE NAVALÓN, VEZINO DELLA.

BPR. Ms. II/1.876. JUAN DE MENA. Las çinquenta con las glosas [que se nombra la Coronación] (de otra mano).

f. 63r: En quinze dias de dezienbre de $\mathrm{M} \mathrm{CCCC}^{\circ} \mathrm{LXXX}^{\circ}$ VI se acabo de screvyr este libro en la çibdad de / Chinchilla e escryuiolo Marco de Navalon, vezino della. Naualon (rúbrica)

Tipo gráfico: Empieza utilizando una escritura híbrida ( $a$ sin copete, $f$ y $s$ bajo la línea, $s$ finales de doble espira muy cerradas) que termina convirtiéndose en cortesana.

16.- 1487. Tarazona. FERDINANDUS GOMEZ DE AGREDA REUEREDISSIMI IN CHRISTO PATRIS ET DOMINI DOMINI ANDREE MARTINEZ, MISERATIONE DIUINA EPISCOPI TIRASONENSIS, HOC PONTIFICALE SUO IUSSU ET IMPENSA TIRASONE SCRIPSI.

BPR. Ms. II/3.051. Pontificale.

f. 50v: Ego Ferdinandus Gomez de Agre/da reueredissimi in Christo patris et domini domini / Andree Martinez, miseratione / diuina episcopi Tirasonensis, hoc pon/tificale suo iussu et impensa / Tirasone scripsi anno Domini $\mathrm{M}^{\circ}$ /CCCC ${ }^{\circ}$ XXXVII $^{\circ}$.

Tipo gráfico: Littera textualis en su variedad rotunda. Bellamente iluminado. En el margen inferior del f. 2r: escudo episcopal.

Procedencia: f. 1v: «De la casa professa de la Compañía de Jesús de Sevilla». «[Sello]: Librería del colegio D.S.H., IHS». Procede de la biblioteca de Francisco de Bruna.

Termina aquí una aportación más al proyecto que tengo iniciado desde hace tiempo con la finalidad de, una vez recopilados el mayor número de colofones con la mención del copista y fecha, establecer su profesión o estatus y ver qué tipo de libros copian y qué tipo gráfico utilizaron para ello. Sin embargo, ante la imposibilidad de verlos todos personalmente, he utilizado los catálogos y repertorios en los que ya están recogidos muchos de ellos, un sinnúmero de fotocopias y la digitalización, caso de existir esa posibilidad. El examen, sin duda, podría haber sido más extenso, dadas las noticias suministradas, pero problemas de edición me han obligado a resumirlos y reseñar las que he creído más interesantes, así como rechazar la relación de catálogos y repertorios utilizados. 